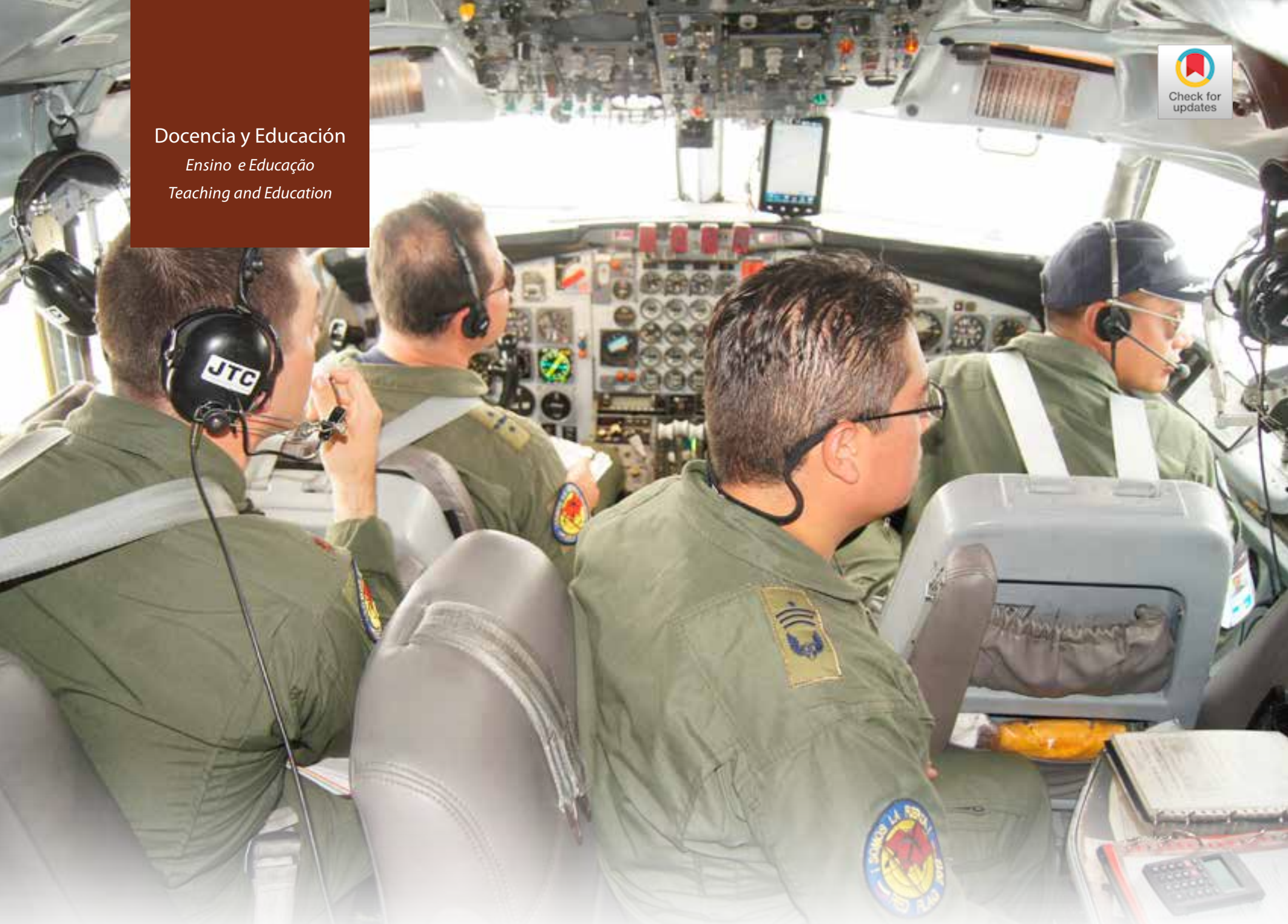

\title{
SIMULADORES DE VUELO: UNA REVISIÓN ${ }^{1}$
}

FLIGHT SIMULATORS: A REVIEW

SIMULADORES DE VÔO: UMA REVISÃO ${ }^{3}$

Luis Carlos Villamil Rico ${ }^{4}$

Edna Joydeth Avella Rodríguez ${ }^{5}$

Jorge Antonio Tenorio Melo ${ }^{6}$

Escuela Militar de Aviación. Bogotá, Colombia.

Universidad del Valle. Cali, Colombia.

\section{CIENCIA Y PODER AÉREO}

ISSN 1909-7050 / E- ISSN 2389-9468 / Volumen 13 / Número 2 / julio-diciembre de 2018 / Colombia /pp. 138-149 Recibido: 25/06/2018

Aprobado par evaluador: 26/08/2018

Doi: 10.18667/cienciaypoderaereo.606 
Para citar este artículo:

Villamil, L. C., Avella, E. J., y Tenorio, J. A. (2018). Simuladores de vuelo: una revisión. Ciencia y Poder Aéreo, 13(2), 138-149. doi: 10.18667/cienciaypoderaereo.606

\begin{abstract}
'Arículo de revisión asociado al proyecto titulado "Desarrollo y construcción de un entrenador de vuelo de la aeronave Cessna T-41 para la Escuela Militar de Aviación Marco Fidel Suárez", financiado por la Convocatoria para el apoyo a proyectos CTel para la investigación formativa de la Fuerza Pública, desarrollada por el Ministerio de Defensa Nacional.
\end{abstract}

${ }^{2}$ Review article associated with the project entitled "Development and construction of a flight trainer for the Cessna T-41 aircraft for Marco Fidel Suárez Military Aviation School', funded by the Call for the support of CTel projects for the formative research of the Public Force, developed by the Ministry of National Defense.

${ }^{3}$ Artigo de revisão associado ao projeto intitulado "Desenvolvimento e construção de um instrutor de vôo para a aeronave Cessna T-41 para a Escola de Aviação Militar Marco Fidel Suárez", financiado pelo Edital de apoio aos projetos de CTel para a pesquisa formativa do Força Pública, desenvolvida pelo Ministério da Defesa Nacional.

${ }^{4}$ Especialista en investigación educativa, ingeniero mecánico, jefe sección investigación Escuela Militar de Aviación. Bogotá, Colombia. Correo electrónico: Ivillamilr@hotmail.com

${ }^{5}$ Maestría en ingeniería con énfasis automatización industrial, ingeniero electrónico, docente Universidad del Valle. Cali, Colombia.

${ }^{6}$ Maestría en ingeniería con énfasis en automatización industrial, especialización en ingeniería con énfasis en automatización industrial, ingeniería electrónica, docente Universidad del Valle. Cali, Colombia.
Resumen: Los simuladores de vuelo permiten realizar maniobras de aprendizaje que en una aeronave real no se podría, ya que se pueden analizar desde fallas humanas hasta las de la aeronave. Este artículo presenta el desarrollo de un estado del arte para el proyecto titulado "Desarrollo y construcción de un entrenador de vuelo de la aeronave Cessna T-41 para la Escuela Militar de Aviación Marco Fidel Suárez", financiado por la "Convocatoria para el apoyo a proyectos CTel para la investigación formativa de la Fuerza Pública", desarrollada por el Ministerio de Defensa Nacional. Para el desarrollo del artículo se realizó una revisión documental que contiene la clasificación, la historia, arquitectura, características, ventajas y aplicaciones de los simuladores de vuelo o dispositivos de entrenamiento de vuelo. Como resultado a destacar se obtuvo que el proyecto inicialmente planteado era el desarrollo de un entrenador, pero se logró desarrollar un simulador tipo A con un sistema de movimiento con tres grados de libertad. Se concluye que la información suministrada por este artículo apoya a los conceptos básicos para la realización de un simulador de vuelo, dándole la capacidad a la Fuerza Aérea Colombiana en el desarrollo de este tipo de tecnologías.

Palabras clave: aeronave, simuladores de vuelo, plataformas de movimiento, FSTD, FAA, EASA.

Abstract: Flight simulators allow to perform learning maneuvers that in an actual aircraft could not be, since they can be analyzed from human faults to those of the aircraft. This article shows the development of a state of the art for the project entitled "Development and construction of a flight trainer of the Cessna T-41 aircraft for the Marco Fidel Suárez Military Aviation School", funded by the "Call for support to CTel projects for formative research of the Public Force ", developed by the Ministry of National Defense. For the development of the article, a documentary review was carried out containing the classification, history, architecture, characteristics, advantages and applications of flight simulators or flight training devices. As a result, to highlight, it was obtained that the initially proposed project was the development of a trainer, but it was possible to develop a type A simulator with a system of movement with three degrees of freedom. It is concluded that the information provided by this article supports the basic concepts to produce a flight simulator, giving the capacity to Colombian Air Force in the development of this type of technology. A flight simulator, giving the Colombian Air Force the ability to develop this type of technology.

Keywords: aircraft, flight simulators, motion platforms, FSTD, FAA, EASA.

Resumen: Os simuladores de vuelo permitem realizar manobras de aprendizado que não se realizam real no se podría, e que se pueden analizar de fallas humanas hasta las da aeronave. Este artículo apresenta o desenvolvimento de um projeto de arte para o projeto "Desarrollo y construcción de um entrenador de vôo da aeronave Cessna T-41 para a Escuela Militar de Aviação Marco Fidel Suárez", financiado por la "Convocatoria para el apoyo a proyectos CTel para a investigação da Força Pública ", promovido pelo Ministério de Defesa Nacional. Para o desenvolvimento do artigo você pode realizar uma revisão documental que contem a clasificación, a historia, arquitectura, características, ventajas e aplicaciones dos simuladores de vuelo o dispositivo de entrenamiento de vuelo. Como resultado pode ser obtido quando o programa foi implantado no desenvolvimento de um sistema de distribuição de dados, em vez de um tipo de processo. Se concluísse a informação administrada por este artigo apóia os conceitos básicos para a realização de um simulador de vuelo, dá-lhe a capacidade para a Força Aérea Colombiana no desenvolvimento deste tipo de tecnologias.

Palavras-chave: aeronave, simuladores de vôo, plataformas de movimento, FSTD, FAA, EASA. 


\section{Introducción}

Los simuladores de vuelo o dispositivos de entrenamiento de vuelo son usados para la capacitación de los pilotos, desarrollando en ellos habilidades de navegación, maniobra y mantenimiento de los sistemas de las aeronaves. Los simuladores han sido utilizados tantos por las milicias en el mundo como por los civiles, desarrollando nuevas tecnologías y nuevos softwares. Este trabajo de investigación busca aportar a los desarrollos realizados en la Escuela Militar de Aviación (EMAVI) sobre entrenadores de vuelo; a su vez, estas plataformas servirán para aumentar las horas de vuelo y adquirir más destreza por parte de los instructores de vuelo y sus alumnos, sin contar el desarrollo de la ingeniería colombiana, especialmente el de la Fuerza Aérea Colombiana (FAC), en la construcción y planificación de plataformas.

Día a día se ha incursionado en cada detalle de los simuladores de vuelo, hoy en día se realizan grandes investigaciones, como son las plataformas de movimiento que van de un grado de libertad hasta los seis; se han desarrollados nuevos instrumentos tanto de navegación, control, visualización, que hoy en día se cuenta con plataformas que pueden llegar a realizar todas las maniobras hechas por una aeronave real. Por lo anterior, la EMAVI requiere una revisión documental para soportar el desarrollo de un sistema que permita aumentar las horas de entrenamiento de los pilotos de la Fuerza Aérea Colombiana, dando soporte a las diferentes aeronaves utilizadas en la instrucción y entrenamiento de los futuros pilotos. Esta investigación busca dar soporte al conocimiento de simuladores de vuelo, ya que los pilotos adquirirían mayores fortalezas en el manejo de la aeronave, haciendo uso del entrenador de vuelo desarrollado por la EMAVI.

Inicialmente la formulación del proyecto estableció el desarrollo de un entrenador de vuelo de la aeronave CESSNA T-41, para el fortalecimiento de las capacidades de los pilotos, teniendo en cuenta que un entrenador de vuelo brinda la posibilidad de entrenamiento continuo a bajos costos, ya que no requiere gastos en combustible, personal de mantenimiento y otros instrumentos de vuelo. Sin embargo, con el desarrollo del estado del arte se pretende resolver cuál es el alcance que debe tener el dispositivo de vuelo (simulador o entrenador) para la aeronave Cessna T-41 con los recursos obtenidos $(\$ 250.000 .000)$ de la convocatoria, con el fin de fortalecer la instrucción del vuelo en la EMAVI y aumentar las capacidades con que cuenta la Fuerza Aérea Colombiana, contribuyendo al desarrollo del país en un aspecto tan importante como la formación de pilotos y el desarrollo de estos dispositivos.

\section{Simuladores de vuelo ¿Qué son?}

Los simuladores de vuelo son dispositivos que recrean ambientes de vuelo, ya sean con movimiento o no. Generalmente son diseñados a partir de aeronaves que repiten acciones como los sonidos, movimientos, controles de vuelo y reacciones de factores externos (turbulencia, vientos, tormentas, nubosidades entre otros), estos son usados para formación, diseños u otras aplicaciones (Federal Aviation Administration, 2014).

\section{Clasificación}

Según la Federal Aviation Administration (FAA), los simuladores de vuelo son categorizados de tres formas: simuladores de vuelo (FS), dispositivos de entrenamiento de vuelo (FTD o FSTD) y dispositivos de formación de la aviación (ATD) (Bernard, 2012; Aeronáutica Civil, 2015).

Simuladores de vuelo completo (FFS).

Estos simuladores de vuelo tienen la más alta categoría, porque incluyen movimiento y capacidad visual, son los más sofisticados sin realizar un vuelo del sistema real. Los niveles de validación de los simuladores FFS aseguran la aerodinámica, características de vuelo, pilotaje de una determinada marca de aeronave (Bernard, 2012; Aeronáutica Civil, 2015). Los FFS también poseen una clasificación de niveles que van desde la $A-D$, siendo el $D$ la más alta categoría.

La clasificación dada por la FAA a los FFS nivel A: son sistemas de movimiento, se requiere con al menos tres grados de libertad (aviones solamente). Los de nivel B: requiere de tres ejes de movimiento y un modelo aerodinámico de mayor fidelidad que hace nivel A (avión y helicóptero). El nivel C: requiere una plataforma de movimiento con los seis grados de libertad, retardo de transporte inferior (latencia) sobre los niveles A y B, su sistema visual debe tener un campo horizontal fuera de la vista de al menos 75 grados para cada piloto. El nivel D: es el nivel más alto de cualificación, los requisitos son los mismos que el nivel C, con adiciones, el sistema visual debe tener un campo horizontal fuera del mundo de vista de por lo menos 150 grados, se requieren sonidos realistas en la cabina del piloto, así como un número de movimiento especial y efectos visuales (Federal Aviation Administration, 2014; Lawn, The enhancement of a flight simulator system with teaching and reserarch applications, 1998). Los siguientes son algunos simuladores de vuelo: el Sukhoi SuperJet full flight simulator (Gusarov, 2011), y el FFS B (Aerosimulators, 2009) entre otros. 
Dispositivos de entrenamiento de vuelo (FTD).

Los dispositivos FTD o llamados Dispositivos de Entrenamiento de Simulación (FSTD), son dispositivos diseñados para representar la configuración de una aeronave específica, pueden tener cabinas cerradas y visualización. Estos sistemas no siempre llevan movimiento, pero son lo suficientemente sofisticados para capacitaciones y certificaciones. Estos dispositivos se pueden observar en centros de enseñanza, universidades y son utilizados ampliamente para capacitaciones o conocimiento de nuevas tecnologías (Bernard, 2012; Aeronáutica Civil, 2015).

La siguiente es la clasificación dada por la FAA a los FTD de nivel 4: este nivel no requiere de un modelo aerodinámico, pero se requiere del modelado de los sistemas precisos. El nivel 5: requiere la programación aerodinámica y el modelado de sistemas. El nivel 6: tiene programación aerodinámica, es para aviones de modelos específicos, tiene control de movimiento y se requiere cabina física. Nivel 7: es para un modelo específico (helicóptero solamente), tiene la aerodinámica, controles de vuelo y sistemas modelados, sistemas de vibración y tiene un sistema visual (Federal Aviation Administration, 2014; Lawn, The enhancement of a flight simulator system with teaching and reserarch applications, 1998).

\section{Dispositivos de formación de la aviación (ATD).}

Los ATD son dispositivos de entrenamiento que replican la instrumentación de las aeronaves, equipos, paneles y controles, se encuentra en un área cubierta de vuelo o una cabina cerrada de vuelo, incluye el hardware y software para representar la aeronave (Federal Aviation Administration, 2014). Se clasifican en: Dispositivo de Entrenamiento de Aviación Básica (BATD), que es un dispositivo que posee controles físicos y puede tener controles virtuales, los controles deben tener un parecido con los controles reales de la aeronave (Bernard, 2012; Federal Aviation Administration, 2014). El Dispositivo de Entrenamiento de Aviación Avanzada (AATD), posee las mismas características del BATD incluyendo un GPS y un piloto automático (Bernard, 2012; Federal Aviation Administration, 2014).

\section{Arquitectura del simulador de vuelo}

Los simuladores de vuelo o los entrenadores de simulación son dispositivos que integran el software y el hardware, están diseñados con diferentes etapas como lo son: equipos de mando, instrumentación, plataformas de movimiento, lenguajes de programación (Bosh, 2011; Kovacova y Koblen, 2012).

\section{Cabina}

En la cabina de los simuladores de vuelo se tiene el panel de mandos, que está compuesto por la instrumentación de vuelo y navegación, también la instrumentación del panel del motor (Alonso, 2006).

Para la instrumentación de vuelo de algunos simuladores se encuentran dispositivos como el anemómetro o indicador de velocidad, altímetro, horizonte artificial, indicador de la velocidad vertical, indicador de viraje y coordinador de viraje entre otros, dependiendo la clase de simulador se consideran más instrumentos de vuelo. Para la instrumentación de navegación se encuentran el indicador de dirección o rumbo, indicador de Very Higth Frecuency Omnidirectional (VOR), indicador Automatic Direction Finder (ADF). Para la instrumentación del panel del motor se encuentra el indicador de presión de aceite, indicador de la temperatura del aceite, indicador de sobrecalentamiento del cilindro, medidor de temperatura de gases de escape, indicador de la presión de combustible, indicador de flujo de combustible, indicador de cantidad de combustible, tacómetro

\section{Software y herramientas de desarrollo}

El software en los simuladores de vuelo desarrollan entornos ya sean geográficos o de ambientación para recrear una visualización real, uno de los lenguajes utilizados es el desarrollado por Microsoft creado por Scott Wiltamuth y Anders Hejlsberg Csharp, las principales características de este lenguaje de programación es la facilidad de programación, incorpora nuevos desarrollos para aplicaciones y tiene la capacidad de orientación a objetos entre otras virtudes (Microsoft, 2015). XML es un metalenguaje, es decir un lenguaje para definir lenguajes, también se encuentra el lenguaje SDK (SimKit Devices). Algunas herramientas de desarrollo son la plataforma .Net desarrollada por Microsoft.

En el mundo de la informática, diferentes compañías han lanzado sistemas operativos al mercado mundial, tanto programas con licencias para su uso, como programas libres que fomentan la cooperación para obtener más utilidades de la herramienta. Actualmente el sistema operativo más usado es Android, que está proyectado a sistemas operativos de dispositivos móviles, y aunque no hace parte de lo que busca este proyecto, hay que resaltar que es reconocido mundialmente y le ha dado un gran empuje (Statcounter, 2017).

En cuanto al ámbito de sistemas operativos orientados a computadores, se destaca en primer lugar a Windows con un $53 \%$, luego Ubuntu con un $39 \%$ y posterior Mac con un $10 \%$, el primero desarrollado por Microsoft, el segundo desarrollado por Linux y el tercero desarrollado por Apple, y aunque la marca mundialmente conocida ha sido Windows, los otros sistemas operativos han tenido un fuerte empuje en los últimos años (Education IT, 2017). 


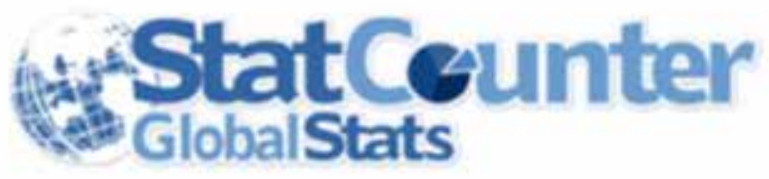

\section{OS Market Share Worldwide March 2012 - March 2017 \\ Android $=$ Windows $=$ ios $=05 x$}

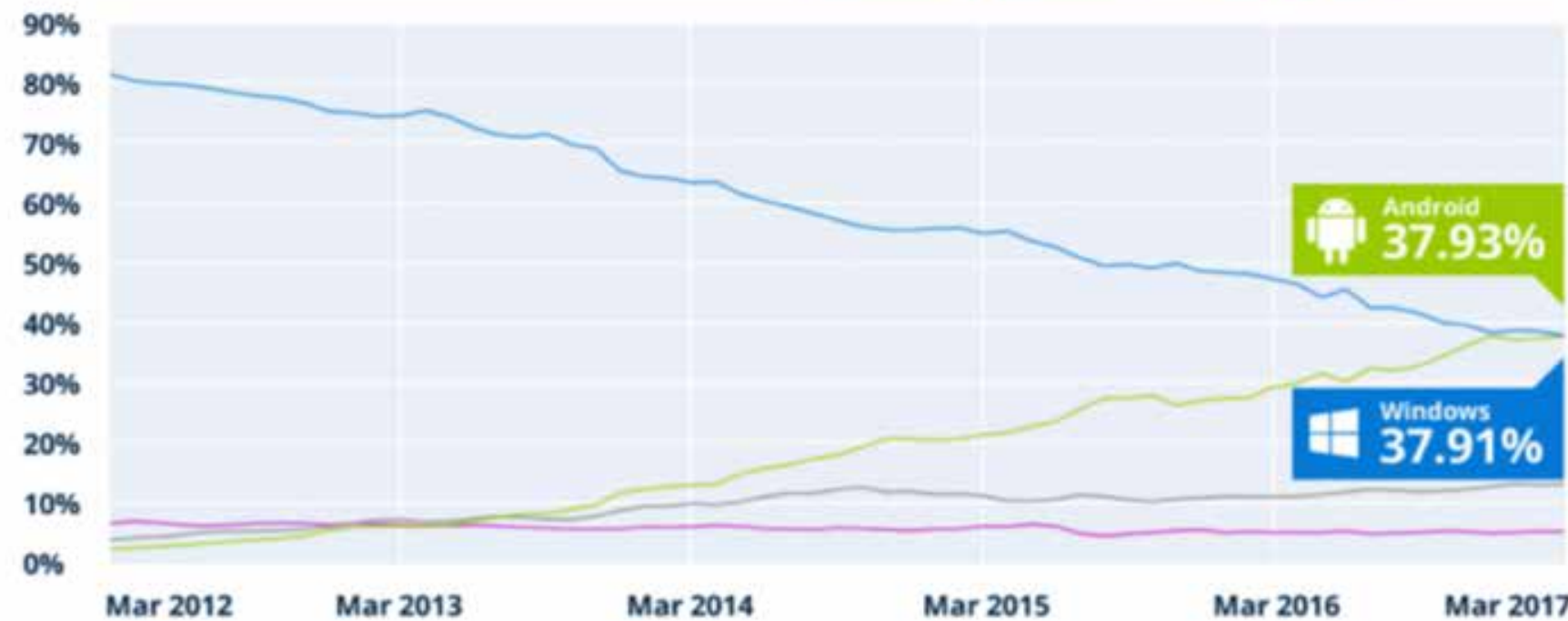

Figura 1. Uso de los sistemas operativos

Fuente. Statcounter, 2017

Los simuladores de vuelo constantemente presentan notables mejoras cada vez que sale una versión nueva de los motores de simulación, los cuales buscan crear entornos virtuales más realistas; a continuación se relacionan los tres más importantes motores de simulación alrededor del mundo, que por sus capacidades se han destacado frente a otros (Vix, 2017):

- Microsoft Flight Simulator (versión 2016).

- X-Plane (versión 11).

- FlightGear.

De los anteriormente mencionados, se destaca el XPlane, porque es un motor preciso basado en el cálculo del efecto del flujo de aire sobre las superficies de los aviones simulados con la utilización de un túnel de viento virtual, adicionalmente, posee certificado de la FAA para entrenamiento de pilotos por instrumentos (X-plane, 2017).

\subsection{Reglamentación}

Los simuladores de vuelo y FSTD son diseñados, producidos, instalados y su evaluación, calificación y aprobación se hace con las normas, criterios y requisitos de la legislación de la aviación, se especifica principalmente en los documentos pertinentes de la OACl, EASA/regulación JAR (en Europa), la regulación FAR (en EE. UU.), de acuerdo con el nivel requerido de certificación.

\section{Historia de los simuladores de vuelo}

En 1903 Orville y Wilbur Wright vuelan por primera vez, con esta primera hazaña se avanza en la aviación, en los siguientes años se desarrollan nuevas tecnologías dando paso a los simuladores de vuelo, los cuales eran utilizados, inicialmente, para la formación de los pilotos, conociendo las partes de la aeronave, los primeros pilotos aprendían progresando a través de una secuencia gradual de ejercicios en un avión real. Después el estudiante progresa a una máquina de mayor potencia, haciendo saltos cortos utilizando el control de elevador y finalmente alcanzar el vuelo. Una variación de este método conocido como el "sistema del pingüino", se realiza utilizando un avión Landborne de envergadura reducida que fue desarrollado durante la Primera Guerra Mundial. Este método se utilizó también en el francés Ecole de Combate, con un monoplano Bleriot Cortado (Ray, 2000; Slob, 2008).

De acuerdo con los desarrollos anteriores, nacen nuevas ideas para los simuladores de vuelo, uno de los dispositivos de esta naturaleza fue el The Sanders "Teacher" en 1910, según Haward

La invención, por lo tanto, de un dispositivo que permitirá al principiante obtener una concepción clara del funcionamiento del control de un avión y de las condiciones existentes en el aire, sin ningún riesgo personal o de otro tipo es bienvenido sin ninguna duda. (1910, p. 1006) 
El Sanders fue construido de componentes reales de aeronaves, fue un avión montado en una junta universal en una posición expuesta y de cara al viento, otros dispositivos de este tipo de simuladores de vuelo fueron las de Eardley Billings, así como el Captive Monoplane (Ray, 2000; Rodríguez, 2014).

Estos simuladores evolucionan sustituyendo los operadores humanos tipo Antoinette, con máquinas de actuadores mecánicos o eléctricos vinculados a los controles del simulador. Uno de los prototipos utilizados son los dispositivos descritos por los franceses Lender y Heildelberg en 1917. Esta realizaba movimientos pitch, roll y yaw por motores de aire comprimido, se incluye el ruido del motor y una visualización. En 1920 nace la necesidad de capacitar a los pilotos de las aeronaves, así que surgen dos enfoques: la capacitación de instrumentos que se hacía sobre aeronaves no móviles para el reconocimiento de la instrumentación; y los links que son equipados con la instrumentación y medios de activación (Ray, 2000).

Uno de los simuladores más conocidos es el Link Trainer, desarrollado por Edwin Link en el periodo de 19271929, patentando en 1930, donde realiza una bomba de succión de accionamiento eléctrico montado en una base fija que alimenta las diversas válvulas de control operados por el timón, mientras que otro dispositivo es accionado por un motor que produce una secuencia repetida de perturbaciones de actitud, este dispositivo se reproduce en paises como Inglaterra, Japón, Francia, Alemania y la URSS, siendo la American Airlines la primera aerolínea en comprar un simulador de vuelo para el entrenamiento de sus pilotos. Desde entonces se desarrollan avances tecnológicos aplicando métodos eléctricos y electrónicos, las señales de radio se utilizan para las radioayudas de la navegación. El dispositivo Aeroconstructor, desarrollado por AE Travis es el primer simulador que utiliza ayudas visuales, que simulaba los efectos de partida, el tono y el movimiento del rodillo (Ray, 2000; Zazula, Myszor, Antemijczuk y Cyran, 2013; Angelo, 2000).

Uno de los grandes avances de los simuladores de vuelo es la llegada de las computadoras analógicas en la Segunda Guerra Mundial, desarrollando las ecuaciones de movimiento de vuelo de la aeronave, permitiendo de este modo la simulación de la respuesta a las fuerzas aerodinámicas; por esta razón, el grupo de Establecimiento de Investigación de las Telecomunicaciones (TRE) diseña simuladores de radar sintético (Dummer, 1949).

En 1943 el Dr. Dehmel y Curtiss_Wright Corporation realizan el primer simulador de vuelo (Boeing 377) para una aerolínea comercial, estos dispositivos utilizan tec- nología A. C., con potenciómetros contorneados; con un control utilizando palancas variables y servo controladores para calcular las fuerzas necesarias para la velocidad, este desarrollo tenía un costo de 120.000 f. De este dispositivo se basan nuevas tecnologías como la aeronave L1090 (Ray, 2000).

En 1958 se desarrollan las primeras plataformas de movimiento, dando inicio con un grado de libertad hasta llegar a los seis produciendo el simulador de vuelo B747. En 1960 las computadoras digitales hacen su surgimiento, dando así la posibilidad de nuevos desarrollos en los simuladores de vuelo. Link desarrolla un simulador de vuelo donde utiliza tres procesadores paralelos para el desarrollo de la aritmética, para funciones y una emisora de radio; emplea una memoria tambor (Ray, 2000; Weingarten, 2005).

Otro aporte para los simuladores de vuelo es la proyección de origen o shadowgraph, que sus primeras aplicaciones se realizan en los simuladores de los helicópteros, estos métodos de visualización son producidos por Giranvions Dorand en Francia y por Belfas. Los desarrollos realizados por Link utilizan sistemas anamórficos conocidos como VAMP. Se avanza en el desarrollo de nuevas tecnologías como las imágenes por computador (Ray, 2000).

Con el desarrollo de los simuladores de vuelo también se hace necesario el uso de reglamentos para la fabricación, ya que cada compañía fabricaba los simuladores con diferentes estándares. En la década de 1970 se crea la organización IATA (International Air Transport Association) y se forma el comité de trabajo FSTSC (Flight Simulator Technical Sub-Committee) donde se desarrollan normas tanto para la simulación como para el fuselaje y aviónica, dando credibilidad a las empresas desarrolladoras (Ray, 2000).

En la actualidad, las investigaciones sobre simuladores de vuelo abarcan complejos sistemas de control (Dongsu y Hongbin, 2007; Songshan, Zongxia y Yaoxing, 2015; Mendoza, Vivas y Rodríguez, 2014; Pradipta, Klunder, Weickgenannt y Sawodry, 2013; Chih-Hsien, Devaney y ChungMing, s.f.; Chomachar y Azizi, 2015); plataformas de movimiento (Monsarrat y Gosselin, 2003; Dongsu, Hongbin y Peng, 2009; Barros dos Santos y Oliveira, 2011; Zhang y Yao, 2009); software (donde se ajustan a la geografía de un lugar determinado, sonidos, clima, fallas técnica o humanas) (Virtual insect flight simulator (VIFS): A software testbed for insect flight, 2001).

En la Tabla 1 se muestra una clasificación de los diferentes simuladores de vuelo, teniendo en cuenta la clasificación de la ESA (EASA, 2015), allí se encuentran simuladores de vuelo para helicópteros (Schmaltz, 2010; Airbus 
CIENCIA Y PODER AÉREO | ISSN 1909-7050 | E-ISSN 2389-9468 | Vol. 13 | Núm. 2 | Jul - Dic 2018 | Escuela de Postgrados de la Fuerza Aérea Colombiana | pp $138-149$

Helicopters, 2015) como aeronaves (Australian Goverment Civil Aviation Safety Authority, 2015; Rodríguez, Sampaio,
Aguiar y Buttacoli, 2014; Odegard, Milenkovic y Buttacoli, 2014; Reinholtz, 1999; Odegard, Sliwinski, King y Hart, 2011).

Tabla 1.

Simuladores de vuelo

\begin{tabular}{|c|c|c|c|}
\hline N. ${ }^{\circ}$ & PAíS & TIPO & CLASIFICACIÓN \\
\hline 1 & Qatar & AW-139 & FFS Nivel B \\
\hline 2 & USA & Bell 407 GX & FFS Nivel B \\
\hline 3 & Singapore & AS365-N3 & FFS Nivel B \\
\hline 4 & Canada & DHC6-300 & FFS Nivel B \\
\hline 5 & China & EC225 LP & FFS Nivel B \\
\hline 6 & Malaysia & EC225 LP & FFS Nivel B \\
\hline 7 & UK & Beech King Air B200 & FFS Nivel C \\
\hline 8 & USA & Learjet 55 & FFS Nivel C \\
\hline 9 & USA & Cessna CJ1 (525) & FFS Nivel C \\
\hline 10 & Thailand & ATR42-300 & FFS Nivel C \\
\hline 11 & Thailand & ATR42-300 & FFS Nivel C \\
\hline 12 & South Africa & ATR72-200 & FFS Nivel C \\
\hline 13 & South Africa & ATR42-300 & FFS Nivel C \\
\hline 14 & USA & Cessna CJ1 (525) & FFS Nivel C \\
\hline 15 & Australia & A320-200 & FFS Nivel D \\
\hline 16 & Australia & A330-200 & FFS Nivel D \\
\hline 17 & Azerbaijan & $757-200$ & FFS Nivel D \\
\hline 18 & Azerbaijan & A320-200 & FFS Nivel D \\
\hline 19 & Bahrain & A320-200 & FFS Nivel D \\
\hline 20 & Bahrain & A330-200 & FFS Nivel D \\
\hline 21 & Brunei Darussalam & S92A & FFS Nivel D \\
\hline 22 & Bulgaria & A320-200 & FFS Nivel D \\
\hline 23 & Canada & Global Express & FFS Nivel D \\
\hline 24 & Canada & DHC8-400 & FFS Nivel D \\
\hline 25 & China & A320-200 & FFS Nivel D \\
\hline 26 & Colombia & ATR72-212A (600 version) & FFS Nivel D \\
\hline 27 & Egypt & 737-800W & FFS Nivel D \\
\hline 28 & Egypt & A320-200 & FFS Nivel D \\
\hline 29 & Egypt & A340-300 & FFS Nivel D \\
\hline 30 & Iceland & $757-200$ & FFS Nivel D \\
\hline 31 & India & AS365-N3 & FFS Nivel D \\
\hline 32 & India & A320-200 & FFS Nivel D \\
\hline 33 & Jordan & 737-800W & FFS Nivel D \\
\hline 34 & Jordan & EMB170 & FFS Nivel D \\
\hline 35 & Kenya & 737-800W & FFS Nivel D \\
\hline 36 & Malaysia & AW-139 & FFS Nivel D \\
\hline 37 & New Zealand & A320-200 & FFS Nivel D \\
\hline 38 & New Zealand & ATR72-212A (600 version) & FFS Nivel D \\
\hline 39 & Norway & S92A & FFS Nivel D \\
\hline 40 & Philippines & A320-200 & FFS Nivel D \\
\hline 41 & Qatar & $787-8$ & FFS Nivel D \\
\hline 42 & Qatar & 777-300ER & FFS Nivel D \\
\hline 43 & Russia & A330-200 & FFS Nivel D \\
\hline 44 & Russia & A320-200 & FFS Nivel D \\
\hline
\end{tabular}




\begin{tabular}{|c|c|c|c|}
\hline 45 & Saudi Arabia & $747-400$ & FFS Nivel D \\
\hline 46 & Singapore & 777-300ER & FFS Nivel D \\
\hline 47 & Singapore & 737-800W SFP & FFS Nivel D \\
\hline 48 & South Africa & ATR72-212A & FFS Nivel D \\
\hline 49 & South Africa & ATR42-500 & FFS Nivel D \\
\hline 50 & Sri Lanka & A330-200 & FFS Nivel D \\
\hline 51 & Sri Lanka & A320-200 & FFS Nivel D \\
\hline 52 & Taiwan (Republic of China) & ATR72-212A (600 version) & FFS Nivel D \\
\hline 53 & Thailand & A320-200 & FFS Nivel D \\
\hline 54 & Thailand & A320-200 & FFS Nivel D \\
\hline 55 & Tunisia & A320-200 & FFS Nivel D \\
\hline 56 & Tunisia & A320-200 & FFS Nivel D \\
\hline 57 & Turkey & 737-800W SFP & FFS Nivel D \\
\hline 58 & Turkey & 737-800W & FFS Nivel D \\
\hline 59 & UAE & $737-800$ & FFS Nivel D \\
\hline 60 & UAE & 737-800W SFP & FFS Nivel D \\
\hline 61 & UK & Hawker 750 & FFS Nivel D \\
\hline 62 & UK & Cessna Citation Mustang & FFS Nivel D \\
\hline 63 & USA & Cessna C750 Citation X & FFS Nivel D \\
\hline 64 & USA & Gulfstream V & FFS Nivel D \\
\hline 65 & Bahrain & A330-200 & FTD Nivel 1 \\
\hline 66 & Bahrain & A320-200 & FTD Nivel 1 \\
\hline 67 & Bulgaria & A320-200 & FTD Nivel 1 \\
\hline 68 & Qatar & A350-900 & FTD Nivel 1 \\
\hline 69 & Qatar & A330-200 & FTD Nivel 1 \\
\hline 70 & Thailand & A330-200E & FTD Nivel 1 \\
\hline 71 & Thailand & ATR72-212A (600 version) & FTD Nivel 1 \\
\hline 72 & Turkey & A340-300 & FTD Nivel 1 \\
\hline 73 & Turkey & A320-200 & FTD Nivel 1 \\
\hline 74 & UAE & A340-600 & FTD Nivel 1 \\
\hline 75 & UAE & A380-800 & FTD Nivel 1 \\
\hline 76 & Qatar & $787-8$ & FTD Nivel 2 \\
\hline 77 & Singapore & $787-8$ & FTD Nivel 2 \\
\hline 78 & Singapore & $787-8$ & FTD Nivel 2 \\
\hline 79 & USA & $787-8$ & FTD Nivel 2 \\
\hline 80 & USA & Bell 429 & FTD Nivel 2 \\
\hline 81 & USA & $787-8$ & FTD Nivel 2 \\
\hline 82 & China & EC225 LP & FTD Nivel 3 \\
\hline 83 & Malaysia & EC225 LP & FTD Nivel 3 \\
\hline 84 & Singapore & AS365-N3+ & FTD Nivel 3 \\
\hline 85 & Singapore & AS365-N3 & FTD Nivel 3 \\
\hline 86 & USA & Bell 407 GX & FTD Nivel 3 \\
\hline
\end{tabular}

\section{Ventajas y aplicaciones}

En la invención realizada por Bennington, muestra la posibilidad de utilizar los aviones que han quedado inoperables para que sean utilizados como aeronaves de entrenamiento, aprovechando la instrumentación existente en el aparato. En el trabajo realizado por Pancoe (2002), describe las características del costo de los entrenadores debido a la gran cantidad de instrumentos y controles que sirven para la simulación. De igual forma Marodi (2002), explica que un simulador de avión es una réplica de tamaño natural de una cabina de avión específico, y es capaz de representar la aeronave en operaciones en tierra y en vuelo.

Según el trabajo realizado por Koekebakker (2001), los simuladores pueden ser categorizados según el área 
de aplicación: simuladores de ingeniería, son usados para evaluar las características de los vehículos como estructura aeroelástica y vibración de alta frecuencia amplificada por el piloto, entre otras. Simuladores de investigación, usados principalmente para la interacción y percepción entre el piloto y la aeronave. Simuladores de entrenamiento, usados para entrenar a los pilotos, en ambiente militar son para procedimientos en base fija.

Entre las ventajas de los simuladores se tienen: requerimientos de seguridad, el equipo mantiene las condiciones de operación limitadas, puede exponer al piloto a situaciones inusuales para que pueda enfrentarse a ellas sin ningún daño. Bajos costos: el uso de simuladores es más económico en comparación con la aeronave y puede ser utilizado para que haya un entrenamiento efectivo. Consideraciones ambientales, cada hora que se está en el simulador hace que se disminuya la generación de contaminación debido al no uso de una aeronave (Allerton, 2010).

Los simuladores de vuelo pueden tener dos características que se ajustan a las necesidades del usuario, como lo son: visualización gráfica o comportamiento físico de la aeronave (Gohl y Leutenegger, 2009; Inaba et al., 2006, pp. 2787-2792). De igual forma, se pueden incluir condiciones de falla para realizar los procedimientos adecuados de corrección (Fountain, 2002)

Los simuladores son una herramienta que permite realizar operaciones sin riesgo para los instrumentos, equipos o personal que interviene. Los simuladores de vuelo tienen un papel muy importante, ya que permiten realizar los ajustes correspondientes sin que los nuevos pilotos o los pilotos que tienen una nueva aeronave sufran algún daño (Muñoz, 2012). También se requiere que existan equipos que permitan realizar entrenamiento de mantenimiento, de tal forma que no se pone en riesgo a la tripulación de la aeronave. El desarrollo de trabajos que buscan recrear las condiciones presentadas en los aviones como en Cristofaro, (2014), Reddy y Arun, (2013) y Alonso (2006), incluye características dinámicas de las aeronaves, evaluando su comportamiento estático y dinámico basado en modelos aerodinámicos. Gohl y Leutenegger (2009) estudian el desempeño que se puede obtener con un controlador de un ala cuando es inestable, además de estudiar los criterios de la estabilidad y rendimiento de la aeronave. De igual forma, los trabajos de simulación de modelos de aeronaves son estudiados por Almeida (2007) y Koekebakker (2001). Los simuladores de entrenamiento se encuentran sobre una plataforma que permite obtener varios grados de libertad (Dongsu y Hongbin, 2007; Davliakos y Papadopoulos, 2008). El trabajo desarrollado por Lawn (1998), muestra el enfoque para aplicaciones de investigación y enseñanza.
Empresas dedicadas al servicio de realizar entrenadores de simuladores aéreos (Flight Safety International Simulation, 2011), aseguran que cerca del $69 \%$ de horas de entrenamiento corresponden a las realizadas en un simulador. En el mercado se encuentran entrenadores más especializados (Elbit System Ltd, 2011), que permiten realizar entrenamientos para el vuelo, estaciones de control, planeamiento de misiones, central de manejo del instructor, entre otros. Además, existen simuladores de vuelo con un desarrollo avanzado como el desarrollado por la NASA (2012): SimLabs: Advancing the science of flight. De igual forma, ofrecen una variedad de simuladores de aeronaves que actualmente se encuentran en el mercado, ya sea para el ambiente comercial o militar (Flight Safety International Simulation, 2011). También existen desarrollos que permiten conocer el comportamiento de la tripulación durante un vuelo (Tan, Chen, Van den Boomen y Rauterberg, 2010; Jirgl, Boni y Jaolovecky, 2015).

En Colombia se ha realizado un trabajo de diseño de simuladores de vuelo (Aguirre y Guarnizo, 2008). En el trabajo realizado por Sizza (2014), resalta la importancia de los simuladores aéreos en la Fuerza Aérea Colombiana, también recalca que se deben potenciar y adecuar a los escenarios del entorno nacional.

\section{Conclusiones}

Las investigaciones sobre los simuladores de vuelo o FSTD han venido evolucionando de acuerdo con la tecnología que se ha desarrollado cada día, realizando un mayor número de investigaciones para el nuevo desarrollo tanto de tecnología como de software, teniendo en cuenta las nuevas tendencias del mundo.

La investigación demuestra que las reglamentaciones realizadas para los simuladores de vuelo o FSTD han sido de ayuda a la evolución de estos, ya que han formalizado y reglamentado los simuladores de vuelo a partir de las nuevas tecnologías y nuevos planteamientos teóricos.

A través de la historia se demuestra que los simuladores de vuelo han ayudado a la formación de pilotos, en sus habilidades de maniobrabilidad, desempeño en situaciones de alto riesgo; también han ayudado a la formación de instructores y al mantenimiento de las aeronaves.

Finalmente, con la clasificación obtenida para los diferentes tipos de simuladores de vuelo se logra evidenciar que lo mínimo requerido a desarrollar en el proyecto es obtener un simulador de vuelo completo (FFS) de tipo A, con una plataforma de movimiento con tres grados de libertad, un sistema visual frontal y lateral que brinde las referencias para el aterrizaje y un software que ofrezca el mejor modelo dinámico para la aeronave Cessna T.41. 


\section{Agradecimientos}

En primera instancia al Ministerio de Defensa Nacional por brindar los recursos para la convocatoria para el apoyo al proyecto "CTel para la investigación formativa de la Fuerza Pública 2015. Por otro lado, a los alféreces y cadetes vincu- lados al proyecto, a todo el personal del grupo académico y grupo de vuelos, y directivos de la Escuela Militar de Aviación por el apoyo brindado para el desarrollo del proyecto (figura 2 y 3), el cual tuvo gran aceptación por parte del personal de instructores y alumnos de vuelo, fortaleciendo el entrenamiento y contribuyendo al ahorro institucional.

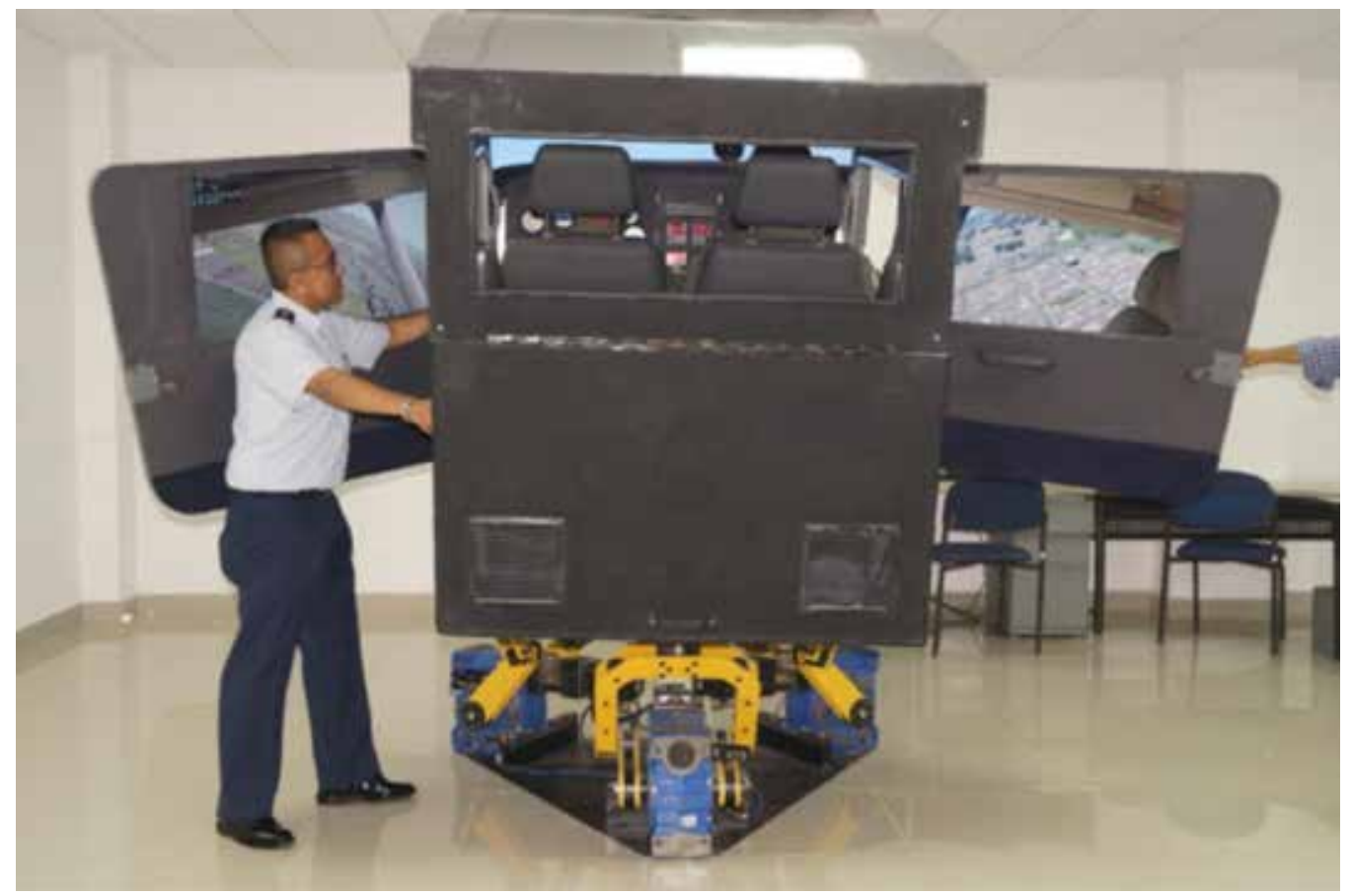

Figura 2. Vista posterior simulador de vuelo T-41 EMAVI Fuente. Prensa EMAVI, 2017

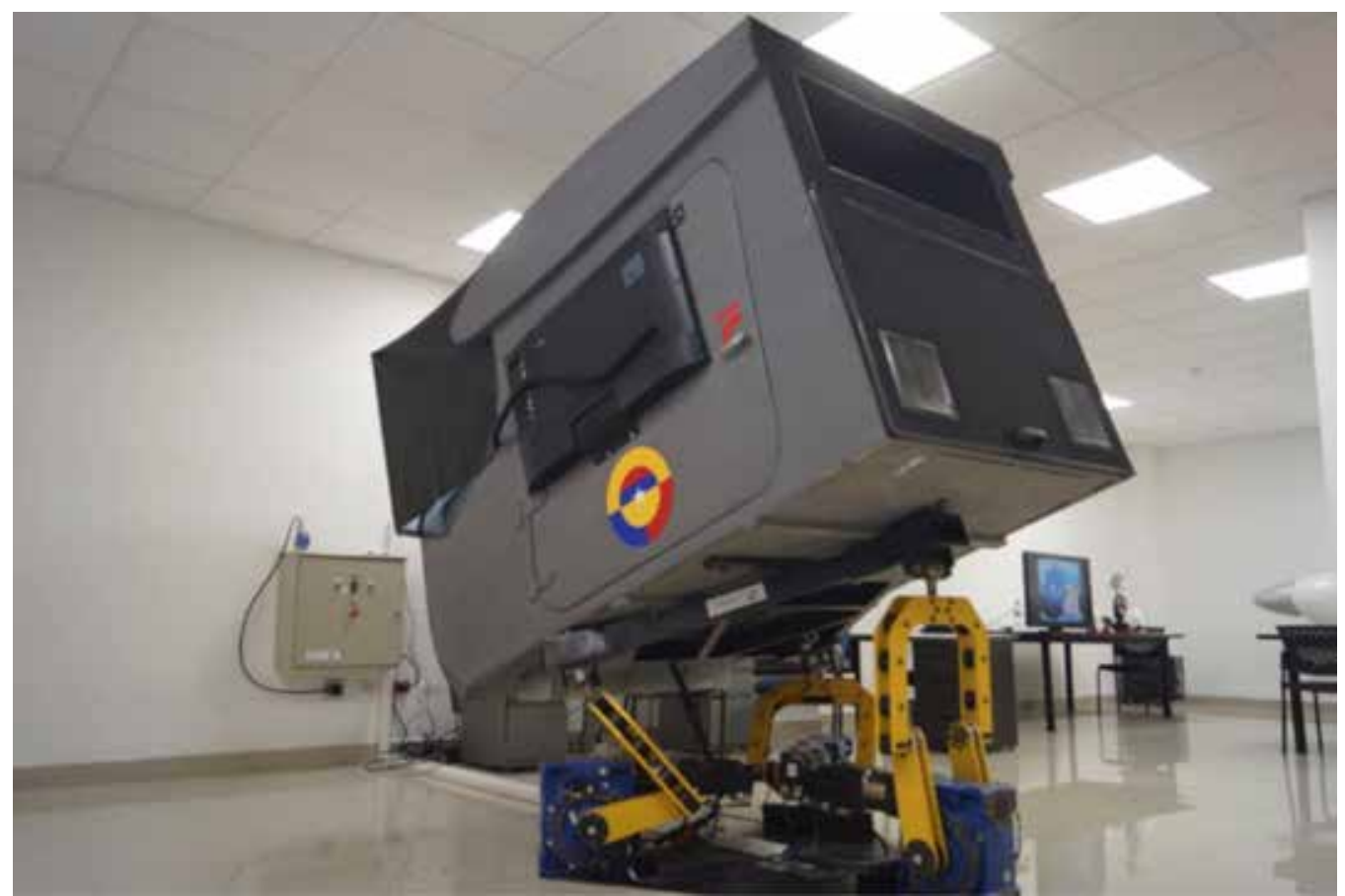

Figura 3. Vista lateral simulador de vuelo T-41 EMAVI Fuente. Prensa EMAVI, 2017 


\section{Referencias}

Aeronáutica Civil. (2015). RAC 24 Dispositivos simuladores para entrenemiento de vuelo. Recuperado de http://www.aerocivil. gov.co/AAeronautica/Rrglamentacion/RAC/Paginas/Inicio. aspx

Aerosimulators. (2009). Flight Training. Recuperado de http:// www.superjetinternational.com/media-center/ssj100-fullflight-simulator-in-venice-achieves-easa-certification/

Aguirre, L., y Guarnizo R., J. (2008). Diseño detallado de un simulador de vuelo dinamico. Bogota D. C.: Universidad de San Buenaventura.

Airbus Helicopters. (2015). Dauphin AS365 N3 / N3 + Full Fligh Simulator. Recuperado de https://www.airbushelicopters.com/ website/en/press/Realistic\%20simulation\%20training\%20 to\%20enhance\%20safety\%20and\%20capabilities\%20of\%20 helicopter\%20search\%20and\%20rescue\%20missions_1651. html

Allerton, D. J. (2010). The impact of flight simulation inaerospace. Recuperado de https://www.aerosociety.com/Assets/Docs/ Publications/DiscussionPapers/The_impact_of_flight_simulation_in_aerospace.pdf

Almeida, D. (2007). UAV Flight Simulator based on ESA Infrastructure Flight simulation models compliant with SMP standard. Lisboa, Portugal: Universidad Técnica de Lisboa.

Alonso, M. (2006). Diseño de una cabina de vuelo virtual. Barcelona: Universidad Politecnica de Catalunya.

Alonso, M. S. (2006). Diseño de una cabina virtual. España: Universidad Politécnica de Cataluña.

Angelo, J. (2000). The link flight trainer. ASME Landmarks, 12.

Australian Goverment Civil Aviation Safety Authority. (2015). Flight simulators and training devices. Australia: AGCASA.

Barros dos Santos, S., \& Oliveira, F. (2011). Longitudinal autopilot controllers test platform hardware in the loop. IEEE International System Conference, 379-386.

Bernard, M. (October, 2012). Real learning throught flight simulatión: The ABCs of ATDs. FAA Saf. Brief, 8-10.

Bosh, M. T. (2011). Diseño de un simulador de helicóptero. España: Universidad Politécnica de Cataluña.

Chih-Hsien, K., Devaney, J., \& Chung-Ming, H. (s.f.). The design of a fuzzy-based adaptive digital controller for a three-degreesof-freedom in-parallel actuated manipulator [for flight simulator]. IEEE, 3, 1328-1332.

Chomachar, A. A., \& Azizi, S. (2015). Design of nonlinear control loader system for a flight simulator (a dynamic inversion approach. IEEE, 1-11.

Cristofaro, M. (2014). Elements of computational flight dynamics for complete aircraft. Southampton. UK: University of Southampton.
Davliakos, I., \& Papadopoulos, E. (2008). Model-based control of a 6-dof electrohydraulic Stewart-Gough platform,. Mech. Mach. Theory, 43(11), 1385-1400.

Dongsu, W., \& Hongbin, G. (2007). Adaptive sliding control of sixDOF flight simulator motion platform. Chinese J. Aeronaut, 20(5), 425-433.

Dongsu, W., \& Hongbin, G. (October, 2007). Adaptive Sliding Control of Six-DOF Flight Simulator Motion Platform. Chinese Aeronaut, 20(5), 294-304.

Dongsu, W., Hongbin, G., \& Peng, L. (2009). Comparative study on dynamic identification of parallel motion platform for a novel flight simulator. IEEE, 2232-2237.

Dummer, G. (1949). Aids to training, the design of radar synthetic training devices for the R.A.F. Proc IEE - Part III Radio Commun, 96(40), 101-115.

EASA. (1 de octubre de 2015). EASA Qualifed FSRDs. Recuperado de https://lisstdis.easa.europa.eu/eqstdis

Education IT. (2017). Sistemas operativos más usados. Centro de capacitación y desarrollo profesional.

Elbit System Ltd. (2011). Aircraft Mission Training Center (MTC).

Federal Aviation Administration. (2014). AC 61-136A. Recuperado de https://www.faa.gov/search/?q=AC+61-136A+-+Federal + Aviation+Administration

Federal Aviation Administration. (2014). Training \& Testing. Recuperado de https://www.faa.gov/training_testing/

Flight Safety International Simulation. (2011). Flight Simulation Training Systems. Broken Arrow.

Fountain, P. J. (2002). USA Patente n. US20030054324A1.

Gohl, F., \& Leutenegger, S. (2009). Aerodynamic performance and stability simulation of different flying wing model airplane configurations.

Gusarov, R. (2011). Sukhoi SuperJet. Recuperado de http://www. ruaviation.com/news/2011/11/22/632/

Haward, D. M. (1910). The Sanders "Teacher". Flight, I/(50), 10061007. Recuperado de https://www.flightglobal.com/pdfarchive/view/1910/1910\%20-\%201009.html

Inaba, Y., Shimada, Y., Uchiyama, K., Abe, K., Ishikawa, Y., Sugimoto, T., \& Abe, A. (2006). Development of flight simulator for humanpowered aircraft the road towards a world record. Sice Icase.

Jirgl, M., Boni, J., \& Jaolovecky, R. (2015). The identification possibilities of the measured parameters of an aircraft model and pilot behavior model on the flight simulator. IEEE Xplore, 1-5.

Koekebakker, S. (2001). Model Based Control of a Flight Simulator Motion System,. Netherlands: Technische Universiteit Deltf.

Kovacova, J., \& Koblen, I. (2012). Selected information on flight simulators - main requirements, categories and their development, production and using for flight crew training in the 
both Slovak Republic and Czech Republic conditions. Incas Bulletin, 4, 73-86.

Lawn, P. (1998). The Enhancement of a Flight Simulator System with Teaching and Research Applications. Texas: University Concordia.

Lawn, P. (1998). The enhancement of a flight simulator system with teaching and reserarch applications. Canada: University Montreal.

Marodi, A. (2002). An improved evaluation method for airplane simulator motion cueing. University of Pittsburgh.

Mendoza, M., Vivas, V., \& Rodríguez, H. (2014). Mechatronic Design, Dynamic Modeling and Results of a Satellite Flight Simulator for Experimental Validation of Satellite Attitude Determination and Control Schemes in 3-Axis. Journal of Applied Research and Technology, 12(3), 370-383.

Microsoft. (2015). Visual C\# Language. Recuperado de https://docs. microsoft.com/en-us/dotnet/csharp/getting-started/introduction-to-the-csharp-language-and-the-net-framework

Monsarrat, B., \& Gosselin, M. (2003). Workspace analysis and optimal design of a 3-leg 6-DOF parallel platform mechanism. IEEE, 19(6), 954-956.

Muñoz, M. (2012). Manuel de vuelo. Recuperado de www.manualdevuelo.com

NASA. (2012). SimLabs: Advancing the science of flight. Recuperado de http://www.simlabs.arc.nasa.gov

Odegard, R., Milenkovic, Z., \& Buttacoli, M. (2014). Model-based GN\&amp;amp;C simulation and flight software development for Orion missions beyond LEO. IEEE Aerospace Conference, $1-13$.

Odegard, R., Sliwinski, K., King, T., \& Hart, J. (2011). Configuring the Orion Guidance, Navigation, and Control flight software for automated sequencing. IEEE Aerospace Conference, 1-13.

Pancoe, E. G. (2002). Motión system for an aircraft flight shmulatur.

Pradipta, J., Klunder, M., Weickgenannt, M., \& Sawodry, O. (2013). Development of a pneumatically driven flight simulator Stewart platform using motion and force control. IEEE. doi: 10.1109/AIM.2013.6584085.

Ray, L. P. (2000). Brief history of flight simulation. SimTec, 11-17.

Reddy, B., \& Arun, P. (2013). Development of real models for aircra$\mathrm{ft}$ simulator. IEEE Xplore, 52-53.

Reinholtz, K. (1999). Applying simulation to the development of spacecraft flight software. IEEE Aerospace Conference, 1, 469476.
Rodríguez, N. J. (2014). Generalidades de los simuladores de vuelo. Tecnoesufa, 21-28.

Rodríguez, R., Sampaio, R., Aguiar, A., \& Buttacoli, M. (2014). FVMS Software-in-the-Loop Flight Simulation Experiments: Guidance, Navigation and Contro. Joint Conference on Robotics, 223-228.

Schmaltz, J. (2010). Flight training simulation. The flight safety multiplie, 21(4), 1-8.

Sizza, J. (2014). Simuladores para entrenamientos en la Fuerza Aérea Colombiana. Ciencia y Poder Aéreo, 9(1), 135-141.

Slob, J. (2008). State-of-the-Art driving simulators, a literature survey. Eindhoven: University of Technology.

Songshan, H., Zongxia, W., \& Yaoxing, S. (2015). Fuzzy robust nonlinear control approach for electro-hydraulic flight motion simulator. Chinese J. Aeronaut, 28(1), 294-304.

Statcounter. (2017). Market Share Worldwide. Recuperado de https://statcounter.com/

Tan, C., Chen, W., Van den Boomen, G., \& Rauterberg, M. (2010). Application of automation for low cost aircraft cabin simulator. Control Autom Syst.

Virtual Insect Flight Simulator (VIFS): A software testbed for insect flight. (2001). Virtual insect flight simulator (VIFS): a software testIEEE International Conference on Robotics and Automation, 4, 3885-3892.

Vix. (2017). Vix.com. ¿Listo para despegar? Los mejores simuladores de vuelo civiles. Recuperado de https://www.vix.com/es/ btg/gamer/62883/listo-para-despegar-los-mejores-simuladores-de-vuelo-civiles

Weingarten, N. (2005). History of in-flight simulation \& flying qualities research at calspan. AIAA Journal of Aircraft, 42(2), 290298.

X-plane. (2017). FAA-Certified X-Plane. Recuperado de https:// www.x-plane.com/pro/certified/

Zazula, A., Myszor, D., Antemijczuk, O., \& Cyran, K. (2013). Flight simulators - From electromechanical analogue computers to moderm laboratory of flying. Adv. Sci. Techol, 7(17), 51-55.

Zhang, Y., \& Yao, Y. (2009). Virtual insect flight simulator (VIFS): A software testbed for insect flight. International Conference on Measuring Technology and Mechatronics Automation, 841 844. 